\title{
PARSING AND INTERPRETING COMPARATIVES
}

Manny Rayner

SICS

Box 1263, S-164 28 KISTA

Sweden

Tel: +4687521500

\section{ABSTRACT}

We describe a fairly comprehensive handling of the syntax and semantics of comparative constructions. The analysis is largely based on the theory developed by Pinkham, but we advance arguments to support a different handling of phrasal comparatives - in particular, we use direct interpretation instead of C. ellipsis. We explain the reasons for dividing comparative sentences into different categories, and for each category we give an example of the corresponding Montague semantics. The ideas have all been implemented within a large-scale grammar for Swedish.

\author{
Amelie Banks \\ UPMAIL \\ Box 1205, S-750 02 UPPSALA \\ Sweden
}

Tel: +4618181051

\section{INTRODUCTION}

This paper is written with two distinct audiences in mind. On the practical side, we try to present a cookbook which the natural language interface implementor can use if he wishes to incorporate comparative constructions into his system's coverage. This is, we trust, interesting in itself; a quick glance at Table 1 should be enough to show that this construction is more common than is perhaps generally realized. Thus in addition to the obvious more, less and as much as, used together with an adjective, adverb or determiner, we also include such words as same, before and after, used in appropriate ways. We also try to give a usable classification of the various kinds of constructions generally lumped together under the blanket heading of "Comparative Ellipsis".

Examples of comparatives

1) John is taller than Mary.

2) Few people run as fast as John.

3) John bought more books than Mary.

4) John was happier in New York than in London.

5) John has more books than Mary has newspapers.

6) John had this job before me.

7) John was born in the same city as Mary.

8) Mary had more friends than John thought.

9) More men than women bought the book.

10) Mary seems brighter than most of the pupils.
Adjectival comparison

Adverbial comparison with "as"

Determiner comparison

Comparison on PP

Clausal comparison

"Before" comparison

"Same" comparison

"S-operator" comparison

Complex comparative determiner

"Simple" phrasal comparison

Table 1 
On the theoretical side, we want to reexamine some fundamental questions concerning the nature of the comparative construction; we are going to argue that our practical work fairly strongly supports a hypothesis that has already appeared in several forms in the theoretical literature, namely that "comparative ellipsis" is a semantic rather than syntactic phenomenon. We expand more on this theme in section 2. In section 3 we present our handling of clausal comparison, which is a straightforward implementation of Pinkham's theory.

The next two sections cover nonclausal comparison, and constitute the main part of the paper. In section 4 we show how Pinkham's predicate copying analysis can be implemented within a Montague grammar framework so that duplication of material is not syntactic copying of parts of the parse-tree but is instead a double application of a higher level function. We demonstrate at length how this method can be used to handle three different kinds of elliptic construction, all of which present problems for the syntactic approach. In section 5 we describe our treatment of the base generated phrasal constructions from section B.2.3 of Pinkham's thesis. (We call these "simple" phrasal comparatives). In the final section we summarize our results; in particular we address ourselves to the question of justifying our classification of comparatives into separate categories instead of providing a unified interpretation.

The current paper is a shortened version of (Rayner \& Banks 88) ("the full paper"), which we will refer to from time to time. This includes among other things test examples and full program listings of a logic grammar based on the SNACK-85 implementation, which covers all forms of comparison discussed here.

\section{PREVIOUS WORK}

The traditional viewpoint has been to explain non-clausal comparatives by means of deletion rules; the first detailed account based on this idea was (Bresnan 73), which strongly influenced most work in the area during the following ten years.

Recently, however, other researchers have pointed out problems with Bresnan's approach; a very thorough and detailed criticism appears in (Pinkham 85) ${ }^{1}$, which has been our main theoretical source. Pinkham gives examples of a wide range of constructions which are difficult or impossible to explain in terms of deletion phenomena, and suggests instead an approach in which at least some comparative constructions are base-generated phrasal and then interpreted using a rule which she calls "distributive copying". The following example 2 shows how the scheme works in practice. Sentence 1a) receives the logical form Ib):

1a) I invited more men than women

1b) I INVITED (MORE $\left[q_{1}\right.$ ( $q_{1}$ men), $q_{2}$ (q2 women)])
1 Hereafter "Pinkham". 
(The object of INVITED is the base generated phrasal). After distributive copying, this becomes 1c):

\section{1c) MORE I $q 1$ (INVITED $q_{1}$ men), $q 2$ (INVITED 92 women)]}

This manoevre, replacing syntactic deletion rules with interpretative copying operations, seems to us very powerful, and (although we formulate it in a rather different way) is one of the central ideas in our own treatment of comparatives. We have in fact taken it even further than Pinkham, who keeps the verb deletion rule of " $\mathrm{C}$ ellipsis" to explain some comparative constructions: in the account presented below in section 4, we get rid of the deletion rules completely and use only interpretative methods.

In this context, it is interesting to look at Levin's LFG-based work on sluicing constructions (Levin 82). Levin presents a variety of arguments to support her claim that sluicing is not a c-structure phenomenon (i.e. not elliptic in nature), but rather explainable at f-structure level (i.e. in some sense related to a semantic copying operation). The differences between sluicing and comparative ellipsis are sufficiently great that this cannot in itself be said to prove anything, but it is none the less indicative of the way in which linguists are thinking about these problems.

In SNACK-85, which uses a framework based on that in (Pereira 83), we perform copying operations on "quanttrees", a level of structure which can loosely be compared with Chomskian logical form or LFG's f-structures.
Viewed in this light, we claim that our treatment of non-clausal comparison (which at first glance might seem somewhat ad hoc) is in fact fairly wellrelated to current tendencies in theoretical linguistics.

\section{CLAUSAL COMPARATIVES}

Most authors are agreed that the case of clausal comparison is the simplest, and for this reason we tackle it first; despite this, it will be seen that there are a few tricky points. Our analysis is heavily based on Pinkham's, and virtually amounts to an implementation of the second section of her thesis; we start by summarizing what we see as the main ideas in her treatment.

The fundamental notion in Pinkham's analysis is to assume that there is an implicit element present in a comparative clause, which is linked to the head of the comparison ${ }^{1}$ in a way similar to that in which a trace or gap is linked to its controller. This "trace" always contains a quantifier-like component. (We will adopt Pinkham's notation and symbolize this as $Q$ ). It may consist of just the $Q$ on its own, or else be an implicit NP composed of the $Q$ together with other material from the head of the comparison.

Pinkham argues that there are essentially three cases; these are exemplified in sentences $2 a$ ) - 2c). In the first of these, just the $Q$ is extraposed; in the second, a $Q$ together with the $\mathrm{CN}$ books, taken from the

1 We endeavour throughout this paper to keep our terminology as close as possible to that used by Pinkham. The terms used are summarized in Appendix 1. 
head more books. If the head contains a comparative adjective, as in $2 \mathrm{c}$ ), then the extra material, consisting of the adjective and the main noun from the head, is obligatory. For a justification, and an explanation of several apparent exceptions, we refer to Pinkham, p. 33 40.

2a) John bought more books than Mary bought $(Q)$ records.

2b) John bought more books than Mary could carry ( $Q$ books).

2c) John bought a more expensive vase than Mary bought (a $Q$ expensive vase).

A scheme of this kind can readily be implemented using any of the standard ways of handling traces. In our system, which is based on Extraposition Grammar (Pereira 83), we use the "extraposition list" to move the material from the head to the place in the comparative clause where it is going to appear; this corresponds to use of the HOLD register in an ATN, or "slash categories" in a GPSG-like framework.

Although this method appears to work well in practice, thre is a theoretical problem arising from the possibility of sentences with crossing extrapositions. We refer to the full paper for further discussion.

\section{DIRECT INTERPRETATION OF NON-CLAUSAL COMPARISON}

\subsection{Basic ideas}

Our first implementation (Banks 86) was based on the conventional interpretation of comparatives: all comparatives are explicit or elliptic forms of clausal comparatives, making the analysis of comparison essentially a syntactic process. In (Banks \& Rayner 87) we presented this in outline and then described some problems we had encountered, which eventually caused us to abandon the approach. Briefly, it turned out that the exact formulation of the syntactic copying process was by no means straightforward: there appeared to be a strong parallel with the well-known arguments against the analogous point of view for coordination constructions. (See e.g. (Dowty et. al. 82), p. 271). As an example, we presented sentence 3 )

3) Everyone spent more money in London than in New York.

which is problematic for a reduction account. We suggested instead that the sentence be thought of as being composed of the following components: the initial everyone, the contrasted elements London and New York, and the duplicated part, which could be rendered (roughly) as is a $P$ such that $P$ spent an amount of money _ in _ where _. In a Montaguegrammar-like formalism, this can then be given the following semantic analysis: 


\section{"Montagovian" analysis of comparative}

(spent $(x, y, z)$ is to be read as " $x$ spent amount $y$ in the city $z ")$

1. everyone

2. New York

3. London

4. spent_ in _

5. spent more in _ than in New York

6. spent more in London than in New York

7. everyone spent more in London than in New York $\lambda Q \forall x: \operatorname{person}(x) \rightarrow Q(x)$

$\lambda \mathrm{Q} \exists z:[z=N e w$ York $\wedge \mathrm{Q}(\mathrm{z})]$

$\lambda \mathrm{Q} \exists \mathrm{z}:[\mathrm{z}=$ London $\wedge \mathrm{Q}(\mathrm{z})]$

$\lambda y \lambda \rho \lambda z \lambda x: \operatorname{spent}(x, y, z) \wedge \mathcal{P}(y)$

$\lambda z \lambda x \exists y: \operatorname{spent}(x, y, z) \wedge$

$\exists y^{\prime}:$ spent $\left(x, y^{\prime}\right.$, New York $) \wedge y>y^{\prime}$

$\lambda x \exists y:$ spent $(x, y$, London $) \wedge$

$\exists y^{\prime}$ :spent $\left(x, y^{\prime}\right.$, New York $) \wedge y>y^{\prime}$

$\forall x:$ person $(x) \rightarrow$

[ $\exists y:$ spent $(x, y$, London $) \wedge$

$\exists y^{\prime}: \operatorname{spent}\left(x, y^{\prime}\right.$, New York $\left.) \wedge y>y^{\prime}\right]$

Table 2

The key point is that the syntactic copying of the deletion approach has been replaced by a semantic operation, a double instantiation of a lambdabound form. The following account summarizes how the idea is implemented within the structure of the SNACK-85 system.

Semantic interpretation in SNACK-85 is performed by first converting the parse-tree to an intermediate form, which we call (following (Pereira 83)) a quant-tree. This is then subjected to rewriting rules before being converted into the final logical form. Normally, these rewriting rules formalize socalled scoping transformations; here, we will also use them to describe the interpretation of non-clausal comparison. The basic motivation is the same, namely to remove rules from the grammar which lack syntactic motivation.

We introduce four new kinds of nodes in addition to those defined in (Pereira
83): we call these comparands, comparative-objects, comparisons, and comparison-placeholders. They interact as follows.

(Stage 1) At the syntactic level, we view the comparative object as a constituent in its associated comparative AP; when the parse-tree is transformed into the quant-tree, the AP gets turned into a comparand node, in which there is a comparative-object $s u b$ node representing the comparative object.

(Stage 2) Rewriting rules then move the comparative-object out of the comparand, leaving behind a placeholder. This is a triple consisting of the compared predicate (the adjective, adverb or whatever), and two logical variables (the "linking" variables), which correspond to the lambda-bound variables $y$ and $\boldsymbol{P}$ above.

(Stage 3) The "raised" comparativeobject node is a 4-tuple. It consists of 
- The two variables $y$ and $\mathcal{P}$ (and is thus "linked" to the placeholder through them - hence the name),

- The comparison type (more than, less than, same as etc.)

- The quant subnode which represents the comparand NP or PP.

The rewriting rules move it upwards until it finds a quant node that it can be compared against. At the moment, the only compatibility requirements are that the quant node and the comparative-object's quant subnode not have incompatible case-markings. This could be improved upon; one way would be to define preference heuristics which gave higher priority to comparisons between quant nodes whose variables are of similar type. The result of merging the two nodes is a comparison node, which is a 5-tuple consisting of

- The comparative-object's quant node

- The quant node it has been merged with

- The comparison type

- The two "linking variables", $y$ and $P$

When the quant-tree is converted into logical form, there should thus be only comparison nodes and placeholder nodes left, with the placeholders "below" the comparisons. In the final stage, the portion of the quant-tree under the comparison node is duplicated twice, and the linking variables instantiated in each copy in the manner described above. So in the "inner" copy, $\boldsymbol{P}$ gets instantiated to a a form $\lambda y: \operatorname{comp}\left(y, y^{\prime}\right)$, where $\operatorname{comp}$ is the type of comparison and $y$ and $y^{\prime}$ are the degree variables; in the "outer" copy, $\boldsymbol{P}$ is instantiated to the value of the inner form.

In the next two subsections, we go further to show how a similar analysis can be used to assign a correct semantics to two other kinds of comparative construction without any recourse to C-ellipsis.

\subsection{Comparatives with "s-operators"}

In this section, we are going to examine comparative constructions like those in 4a), 4b) and 4c). These have a long and honourable history in the semantics literature; $4 \mathrm{c}$ ) is a famous example due to Russell.

4a) Mary had more friends than John had expected.

4b) Most people paid more than Mary said.

4c) John's yacht was longer than I thought.

In order to handle examples like these within our framework, we need a syntactic representation which does not involve ellipsis. Our solution is to introduce a syntactic constituent which we call an "s-operator": we define this implicitly by saying that an "soperator" and a sentential complement combine to form a clause. ${ }^{1}$ Thus the italicized portions of the sentences above are deemed to be s-operators, and in each of them the s-operator's

1 In a categorial grammar framework like HPSG (Pollard \& Sag 88), we could simply identify an soperator with a constituent of the form S/S-COMP. It is fairly straightforward to define s-operators in XG-grammar. 
missing complement is viewed as a kind of null pronoun.

Although this move may in English seem syntactically quite unmotivated, there are other languages where evidence can be found to support the claim that these pronouns really exist. In Russian, where comparative constructions very closely follow the English and Swedish patterns, they can optionally appear in the surface structure as the pronoun $3 T 0$. The following sentence illustrates this.
Он купил больше книт чем я это He bought more books than I это

думал.

thought.

Semantically, the analysis of such sentences is exactly parallel to that in the preceding subsection. Comparing 4b) with 3), the "initial part" is most people, and the "contrasted elements" are the s-operator Mary said and an implicit trivial s-operator which we can write as (it is true that). The "duplicated part" is the predicate is a $P$ such that $P$ paid _ amount of money where _. Wē can sketch a "Montagovian" analysis similar to that in table 2

\section{"Montagovian" analysis of s-operator comparative \\ (paid $(x, y)$ is to be read as "x paid $y$ amount of money")}

1. most people

2. Mary said

3. (it is true that)

4. paid

5. paid more than Mary said

6. (it is true that) paid more than Mary said

7. most people paid more than Mary said $\lambda Q: \operatorname{most}(\lambda x: \operatorname{person}(x), Q)$

$\lambda Q: \operatorname{said}(m, Q)$

$\lambda Q: Q$

$\lambda y \lambda \rho \lambda x: \operatorname{paid}(x, y) \sim P(y)$

$\lambda x \exists y$ paid $(x, y) \wedge$

$\exists y^{\prime} \operatorname{said}\left(m, p a i d\left(x, y^{\prime}\right) \wedge y>y^{\prime}\right)$

$\lambda x \exists y$ paid $(x, y) \wedge$

$\exists y ' \operatorname{said}\left(m, p a i d\left(x, y^{\prime}\right) \wedge y>y^{\prime}\right)$

$\operatorname{most}(\lambda x$ :person $(x)$,

$$
\begin{gathered}
\lambda x: \exists y: \operatorname{paid}(x, y) \wedge \\
\quad \exists y y^{\prime s a i d}\left(m, p a i d\left(x, y^{\prime}\right) \wedge\right. \\
\left.y>y^{\prime}\right)
\end{gathered}
$$

Table 3

The implementation of this analysis in terms of quant-tree rewriting rules involves only a slight extension of the method described in section 4.1 above.
The reader is referred to the program code in the full paper for the concrete details. 


\section{3. "Parallel" phrasal comparatives}

Comparative constructions of the type illustrated in $5 \mathrm{a}$ ) have been the object of considerable controversy. The orthodox position was that they were "parallel" constructions: 5a) would thus be a reduced form of $5 b$ ).

5a) More women than men read "House and Garden".

5b) More women read "House and Garden" than men read "House and Garden".

Pinkham, however, gives good reasons for supposing that this is not the case, and that the construction is in some sense base generated phrasal (p.121123). It will presumably not come as a revelation to hear that we agree with this idea, though we express it in a somewhat different way.

Our interpretation of Pinkham's analysis recasts the more ... than... construction as a special kind of determiner. We introduce an extra rule for NP formation: in addition to the normal $\mathrm{NP} \rightarrow$ Det $+\mathrm{CN}$, we also have $\mathrm{NP} \rightarrow$ CompDet $+\mathrm{CN}+\mathrm{CN}$. (The details can be found in the full paper). This allows us as usual to give the constituent structure without use of ellipsis, and then to interpret it using a suitable predicate-copying operation.

Once again we illustrate with a Montague-style example.

"Montagovian" analysis of "parallel" phrasal comparative

$(\operatorname{reads}(x, y)$ is to be read as " $x$ habitually reads $y ")$

1. women

2. men

3. more

4. more women than men

5. "House and Garden"

6. read "House and Garden"

7. more women than men read "House and Garden" $\lambda x: \operatorname{woman}(x)$

$\lambda x: \operatorname{man}(x)$

$\lambda P \lambda Q \lambda R:$ more $(P, Q, R)$

$\lambda R$ : $\operatorname{more}(\lambda x:$ women $(x), \lambda x: \operatorname{men}(x), R)$

$\lambda x: x=$ "H \& G"

$\lambda x: \operatorname{read}(x, y) \wedge y=" H \& G "$

$\operatorname{more}(\lambda x$ : $\operatorname{women}(x), \lambda x: \operatorname{men}(x)$, $\lambda x: \operatorname{read}(x, " H \& G "))$

Table 4

It is interesting to compare our treatment with that suggested in (Keenan \& Stavi 86) (p.282-284) for comparative adjectival constructions like that in 6a); they argue convincingly that these are to be regarded as directly interpreted, rather than as "reduced forms" of sentences like 6b). It seems to us that their arguments can be adapted to support the analysis of "parallel" phrasals given above; so if we were to extend their example, we would have that $6 \mathrm{~b}$ ) in its turn was also to be interpreted directly, rather than considered a reduction of $6 \mathrm{c}$ ).

6a) More male than female students passed the exam. 
6b) More male students than female students passed the exam.

6c) More male students passed the exam than female students passed the exam.

\section{5 "SIMPLE" PHRASAL COMPARATIVES}

We finally turn our attention to a third type of comparative construction, which does not properly seem to fit into any of the patterns given above. We start by giving in 7) - 9) some examples of the kind of sentence we have in mind.

7) Mary seems brighter than most pupils.

8) He ran faster than the world record. ${ }^{1}$

9) John needs a bigger ${ }^{2}$ spanner than the No. 4.

Pinkham uses constructions like these as her key examples when demonstrating the existence of basegenerated phrasal comparatives. Looking for instance, at 9), we claim with Pinkham that the most natural solution is to treat bigger spanner than the No. 4 as a closed constituent with a semantic interpretation which does not involve the rest of the sentence.

It may not be obvious at first why this should be so, and we pause briefly to examine the possible alternatives. Firstly, suppose that we tried to use a reduction/predicate copying account. This would make 9) a form of 9a):

${ }_{1}^{1}$ Pinkham's example 124a, p. 136

2 We will treat "bigger" as though it were actually "more big" for the usual reasons. 9a) John needs a (big to extent $X$ ) spanner, $X$ such that John needs the (big to extent $Y$ ) No. 4. spanner, $X>Y$.

implying that John needs the No. 4. This is clearly wrong; the "needs" isn't copied in any way, and in fact the scope of any copying operation must be limited to the phrase bigger spanner than the No. 4. If we are absolutely bent on using copying, it appears to us that the only way in which it can be done is to treat 9) as derived from 9c) through 9b)

9b) John needs a spanner which is bigger than the No. 4.

9c) John needs a spanner which is (big to extent $X), X$ such that the No. 4 is (big to extent $Y$ ), $X>Y$.

To be honest, we can't completely discount this approach. However, since it makes bigger than the No. 4 into a constituent in the intermediate $9 \mathrm{~b}$ ), we think it simpler to interpret the phrase structure directly, as is illustrated in the following Montagovian analysis. 


\section{Montagovian analysis of "simple" phrasal comparative}

(needs $(x, y)$ to be read as " $x$ needs something of which the predicate $y$ holds")

1. John

2. needs

3. No. 4

4. big

5. spanner

6. the

7. more

8. more big than the No. 4

9. a bigger spanner than the No. 4

10. John needs a bigger spanner than the No. 4 $\lambda x: x=$ John

$\lambda x, y:$ needs $(x, y)$

$\lambda x$ : type_of $(x$, No. 4$)$

$\lambda x, y: \operatorname{big}(x, y)$

$\lambda x$ : spanner $(x)$

$\lambda \mathrm{P} \lambda \mathrm{Q}:$ the $(\mathrm{P}, \mathrm{Q})$

$\lambda P \lambda Q \lambda R:(\lambda x: \exists y: P(x, y) \wedge$

$R\left(Q, \lambda z: \exists y^{\prime}: P\left(z, y^{\prime}\right)\left(y>y^{\prime}\right)\right)$

$\lambda x: \exists y: \operatorname{big}(x, y) \wedge$

the( $\lambda z$ : type_of $(x$, No. 4),

$\left.\lambda z: \exists y^{\prime}: \operatorname{big}\left(z, y^{\prime}\right) \wedge\left(y>y^{\prime}\right)\right)$

$\lambda x:$ spanner $(x) \wedge$

$\exists y: \operatorname{big}(x, y) \wedge$

the ( $\lambda z$ : type_of $(x$, No. 4$)$,

$\left.\lambda z: \exists y^{\prime}: \operatorname{big}\left(z, y^{\prime}\right) \wedge\left(y>y^{\prime}\right)\right)$

needs(John,

$\lambda x:$ spanner $(x) \wedge$

$\exists y: \operatorname{big}(x, y) \wedge$

the ( $\lambda z$ : type_of $(x$, No. 4$)$,

$\left.\lambda z: \exists y^{\prime}: \operatorname{big}\left(z, y^{\prime}\right) \wedge\left(y>y^{\prime}\right)\right)$

Table 5

It will be apparent that bigger than the No. 4 turns up as a constituent here too, and thus our solution is in a sense equivalent with the alternate one proposed above. This is a striking illustration of the difficulties that can attend any efforts to make rigorous comparisons between different syntactic-semantic analyses of naturallanguage constructions.

\section{CONCLUSIONS}

We have presented a method for syntactic and semantic interpretation of comparative sentences. This has been done by dividing our material into three separate groups, each of which are treated differently:

- Clausal comparatives (section 3), which are handled by extraposing a constituent containing a $Q$, following Pinkham's theoretical analysis. 
- Phrasal comparatives (section 4), treated by direct interpretation using "predicate copying".

- "Simple" phrasals (section 5), handled by a different direct interpretation method.

We do not claim that this classification is the only way to explain the facts; as we have said above, it would be possible to rewrite simple phrasal comparatives into directly interpreted phrasal comparatives, and also to rewrite directly interpreted phrasal comparatives as clausal comparatives. We think, however, that this manoevre would give us nothing in the form of real gains; even though a unified solution might seem more elegant, the syntactic transformations needed are more complicated than the use of different categories. Thus our first argument against a unified approach is the practical one: we need do less work as implementors if we adopt the classification described here. Despite this, we suspect that many readers (especially those more theoretically than practically inclined) would find it comforting to have some direct evidence that supports our point of view. In this connection we think that the following data from Swedish may be of interest.

Comparative constructions in Swedish are virtually identical to the corresponding ones in English. One significant difference, however, is the distribution of the relative pronoun vad ("what"); this can optionally be inserted after the comparative marker in some constructions, as shown in 10) and 11$)^{1}$.

10) Johan köpte fler böcker än John bought more books than

(vad) Maria gjorde. (what) Mary did.

11) Johan har ett dyrare John has a more expensive hus än (vad) jag har.

Given the correspondences between clausal comparison and relative clauses described in section 4 , it is very tempting to account for the "vad" as a relative pronoun realizing the normally null $Q$. If we are prepared to accept this, it then appears significant that "vad" may not be used in most phrasal comparatives, as shown in 12) and 13). This would seem problematic for a transformational account, but is quite natural if phrasal comparatives are treated by direct interpretation; there isn't any $Q$, so it can't be realized as a "vad".

14) Johan köpte fler böcker än John bought more books than

("vad) Maria.

(*what) Mary.

15) Fler kvinnor än ("vad) män More women than ("what) men

läser "Hänt i Veckan". read "News of the World".

There is, however, one exception to the rule: "vad" may appear in the "s-

${ }^{1}$ This is also possible in some dialects of English. 
operator" constructions from section 5.1 above, as shown in 16).

16) Johan köpte fler böcker än John bought more books than

(vad) Maria trodde. (what) Mary thought.

We are not certain how to explain this, and leave the reader to judge the facts for himself ${ }^{1}$; but despite this irregularity, we think the other data gives our theory a considerable amount of concrete backing.

\section{APPENDIX: TERMINOLOGY}

Comparative Clause: the clause introduced by the comparison marker.

Compared Element: the largest constituent in the main or the comparative clause, the leftmost element of which is a comparison marker or the comparative quantifier Q.

Comparison Marker: words like than, as, before, after.

Head of the Comparison: refers to the compared element in the main clause.

Phrasal Comparative: a comparative complement which appears to be the reduced form of a comparative clause. This may be a remnant of the application of Comparative Ellipsis to a

1 One possibility is that this is a result of cognitive limitations in the human sentence-processing mechanism, since an arbitrary amount of text can separate a "vad" from the realization that the construction is s-operator rather than clausal comparison. comparative clause, or it may be base generated.

Q: An (implicit or explicit) comparison quantifier which is extraposed in the interpretation of clausal comparatives.

\section{REFERENCES}

(Banks 86) Banks, A. Modifiers in Natural Language, Bachelor's Thesis, Uppsala University, 1986.

(Banks \& Rayner 87) Banks, A. and Rayner, M., Comparatives in Logic Grammars - Two Viewpoints, Proceedings of the 2nd International Workshop on Natural Language Understanding and Logic Programming, p. 131 137, 1987

(Bresnan 73) Bresnan, J. Syntax of the Comparative Clause Construction in English, Linguistic Inquiry 4, p. 275-343, 1973

(Dowty et al. 82) D. Dowty, R.E. Wall and S. Peters, Introduction to Montague Semantics D. Reidel, 1982

(Keenan \& Stavi 86) Keenan, E.L and Stavi J. Natural Language Determiners, Linguistics and Philosophy 9, p. 253-325

(Levin 82) Levin, L., Sluicing: A Lexical Interpretation Procedure, in Bresnan, J. (ed.) The Mental Representation of Grammatical Relations, MIT Press, 1982

(Pinkham 85) Pinkham, J. The Formation of Comparative Clauses in French and English, Garland Publishing Inc, New York, 1985

(Pereira 83) Pereira, F.N.C. Logic for Natural Language Analysis, SRI Technical Note No 275, 1983

(Pollard \& Sag 88) C. Pollard and I. Sag, Information-based Syntax and Semnantics, Vol. 1, CSLI, 1988

(Rayner \& Banks 86) Rayner, M. and Banks, A. Temporal Relations and Logic Grammars, Proceedings of ECAI-86, Vol. 2 p.9-14, 1986 